\title{
ON THE VALIRON DEFICIENCIES OF MEROMORPHIC FUNCTIONS OF FINITE ORDER( $\left.{ }^{(}\right)$
}

\author{
BY \\ D. F. SHEA
}

Introduction. A few years ago, A. Edrei and W. H. J. Fuchs [3] established the following result about the deficient values of a certain class of entire functions.

THEOREM A. Let $f(z)$ be an entire function of finite order $\lambda$, all of whose zeros lie on the negative real axis.

Then if $\lambda>1, f(z)$ has zero as a Nevanlinna deficient value.

This result discloses the remarkable fact that a simple geometrical restriction on the distribution of the zeros of an entire ifunction is almost, by itself, enough to make zero a deficient value.

Since the publication of [3], a number of extensions of Theorem $A$ have been found (cf. for instance [6], [7], [9]). However, none of these results expresses "best possible" relations between the order of the function and the size of its Nevanlinna deficiencies, and to find such relations seems very difficult. It is the purpose of this note to show that, if we replace Nevanlinna's definition of deficiency by the one introduced by Valiron, sharp bounds on these deficiencies can be found for certain classes of meromorphic functions. From these bounds it is possible to deduce, as immediate consequences, several results on the growth of the functions considered.

1. Terminology and notations. We assume that the reader is familiar with the fundamental concepts of Nevanlinna's theory of meromorphic functions and in particular with the most usual of its symbols:

$$
\stackrel{+}{\log }, m(r, f), n(r, f), N(r, f), T(r, f) \text {. }
$$

We shall use, whenever this is possible without ambiguity, the simplified notations $m(r, c), n(r, c), N(r, c), T(r)$ in place of $m(r, 1 /(f-c)), n(r, 1 /(f-c))$, $N(r, 1 /(f-c)), T(r, f)$.

The letters $\lambda$ and $\mu$ denote the order and lower order of $f(z)$, respectively:

Presented to the Society, July 9, 1965; received by the editors September 23, 1965.

(1) This research was sponsored by the Air Force Office of Scientific Research, Office of Aerospace Research, United States Air Force, under AFOSR Grant No. AF-AFOSR 421-63. 


$$
\lambda=\limsup _{r \rightarrow+\infty} \frac{\log T(r)}{\log r}, \quad \mu=\liminf _{r \rightarrow+\infty} \frac{\log T(r)}{\log r} .
$$

Following Valiron, we say that $f(z)$ is of regular growth if $\lambda=\mu$.

The Nevanlinna deficiency $\delta(c, f)$ of the value $c$ for the function $f(z)$ is, by definition,

$$
\delta(c, f)=1-\limsup _{r \rightarrow+\infty} \frac{N(r, c)}{T(r, f)} .
$$

The Valiron deficiency $\Delta(c, f)$ of $c$ is

$$
\Delta(c, f)=1-\liminf _{r \rightarrow+\infty} \frac{N(r, c)}{T(r, f)} .
$$

A value $c$ for which $\Delta(c, f)>0$ is said to be deficient in the sense of Valiron; if $\delta(c, f)>0$, then the value $c$ is deficient in Nevanlinna's sense.

It is clear from Nevanlinna's first fundamental theorem that

$$
0 \leqq \delta(c, f) \leqq \Delta(c, f) \leqq 1,
$$

and, further, that $\Delta(c, f)$ is positive if and only if the asymptotic relation

$$
N(r, c) \sim T(r, f)
$$$$
(r \rightarrow+\infty)
$$

fails to hold. A classical result of Ahlfors-Frostman-Nevanlinna [11, p. 280] implies that (1.3) holds for all complex values $c$ except those belonging to an exceptional set of inner capacity zero.

2. Statement and discussion of results. The asymptotic behavior of the following simple entire function is completely known (cf. [10, p. 18]):

$$
F_{\lambda}(z)=\prod_{n=1}^{\infty}\left(1+\frac{z}{n^{\alpha}}\right) \quad(\alpha=1 / \lambda, 0<\lambda<1) .
$$

In particular, it is easy to verify that $F_{\lambda}(z)$ has order $\lambda$, lower order $\mu=\lambda$, and further that

$$
\lim _{r \rightarrow \infty} \frac{N(r, 0)}{T\left(r, F_{\lambda}\right)}= \begin{cases}1 & \left(0<\lambda \leqq \frac{1}{2}\right), \\ \sin \pi \lambda & \left(\frac{1}{2}<\lambda<1\right) .\end{cases}
$$

Hence

$$
\Delta\left(0, F_{\lambda}\right)=1-\sin \pi \lambda>0
$$

when $1 / 2<\lambda<1$, and thus the value 0 is deficient, for these functions $F_{\lambda}(z)$, in the sense of either of the definitions (1.1), (1.2).

To what extent is (2.1) typical of arbitrary entire functions having only negative zeros and order $\lambda$ ? One answer to this question is contained in 
THeOREM 1. Let $f(z)$ be a meromorphic function of order $\lambda$, whose zeros lie on the negative real axis and whose poles lie on the positive real axis.

Put

$$
X=1-\Delta(0, f), \quad Y=1-\Delta(\infty, f)
$$

Then necessarily

$$
X^{2}+Y^{2}-2 X Y \cos \pi \lambda \leqq \sin ^{2} \pi \lambda
$$

when $1 / 2 \leqq \lambda \leqq 1$.

When $\lambda<1 / 2$, (2.3) still holds provided

$$
X \geqq \cos \pi \lambda \text { and } Y \geqq \cos \pi \lambda \text {. }
$$

Since for entire functions $N(r, \infty) \equiv 0$ and hence $Y=0$, Theorem 1 implies

$$
\Delta(0, f) \geqq 1-\sin \pi \lambda \quad\left(\frac{1}{2}<\lambda \leqq 1\right)
$$

for any entire function $f(z)$ with negative zeros and order $\lambda$, and this is the desired generalization of (2.1).

If the quantities $X, Y$ are regarded as the coordinates of a point in an $X Y$ plane, then the definitions (2.2) and (1.2) show trivially that the point $(X, Y)$ is always confined to the unit square

$$
0 \leqq X \leqq 1, \quad 0 \leqq Y \leqq 1 .
$$

For the functions of Theorem 1 , the point $(X, Y)$ is subject to the additional restriction that it lie inside or on the ellipse

$$
X^{2}+Y^{2}-2 X Y \cos \pi \lambda=\sin ^{2} \pi \lambda,
$$

at least when $\lambda \geqq 1 / 2$.

When $\lambda<1 / 2$, it is not hard to see that $(X, Y)$ may lie outside this ellipse if $X<\cos \pi \lambda$ or $Y<\cos \pi \lambda$, so that the conditions (2.4) are necessary in this case.

That the inequality (2.3) is best possible follows, for orders $\lambda$ less than 1 , from the examples in [8, pp. 116-119]. When $\lambda=1$, Theorem 1 is necessarily sharp since in this case (2.3) asserts

$$
\liminf _{r \rightarrow+\infty} \frac{N(r, 0)}{T(r)}=\liminf _{r \rightarrow+\infty} \frac{N(r, \infty)}{T(r)}=0 .
$$

Combining Theorem 1 with a result of Edrei [1, Theorem 1], we can establish a sort of tauberian theorem connecting the asymptotic behavior of the ratios $N(r, 0) / T(r), N(r, \infty) / T(r)$ with the regularity of growth of a class of functions $f(z)$.

COROLlaRY 1.1. Let $f(z)$ be a meromorphic function of order $\lambda \leqq 1$ and lower order $\mu$, with negative zeros and positive poles.

Assume that the limits 


$$
X=\lim _{r \rightarrow \infty} \frac{N(r, 0)}{T(r)}, \quad Y=\lim _{r \rightarrow \infty} \frac{N(r, \infty)}{T(r)}
$$

exist. If $\lambda \leqq 1 / 2$ make the additional assumption

$$
X<1 \text { and } Y<1 \text {. }
$$

Then, $\lim _{r \rightarrow \infty}(\log T(r) / \log r)$ exists and

$$
X^{2}+Y^{2}-2 X Y \cos \pi \lambda=\sin ^{2} \pi \lambda .
$$

Assume further that $0<\lambda<1$. Then there exists a unique number $\beta$, given explicitly by

$$
\beta=\frac{1}{\lambda} \cos ^{-1}(Y) \quad(0<\beta<\pi),
$$

such that

$$
\lim _{k \rightarrow \infty} f\left(r_{k} e^{i \theta}\right)= \begin{cases}\infty & \text { if }|\theta| \leqq \beta-\varepsilon, \\ 0 & \text { if }|\pi-\theta| \leqq \beta-\varepsilon\end{cases}
$$

holds, uniformly in $\theta$, for any $\varepsilon>0$ and for any sequence $\left\{r_{k}\right\}$ of Pólya peaks $[2, p .81]$ of $T(r, f)$.

In particular, it is easy to see from (2.9) that the rays $\arg z= \pm \beta$ are lines of Julia for the functions of Corollary 1.1.

The next theorem shows that the extreme behavior indicated in (2.5), for functions of order 1 , occurs for entire functions of any positive integral order.

THEOREM 2. Let $f(z)$ be an entire function of finite order $\lambda(>1)$ and lower order $\mu$, all of whose zeros lie on the negative real axis.

Then if $\lambda$ is not an integer, we have

$$
[\lambda] \leqq \mu
$$

(where $[\lambda]$ denotes the greatest integer not exceeding $\lambda$ ), while

$$
\lambda \leqq \mu+1
$$

holds if $\lambda$ is an integer; in either case,

$$
\liminf _{r \rightarrow+\infty} \frac{N(r, 0)}{T(r)} \leqq \min _{\mu \leqq \rho \leqq \lambda} \frac{|\sin \pi \rho|}{1+|\sin \pi \rho|} .
$$

In particular, if either $\mu$ or $\lambda$ is a positive integer, then necessarily

$$
\underset{r \rightarrow+\infty}{\lim \inf } \frac{N(r, 0)}{T(r)}=0
$$

Theorem 2 thus completes a result of I. V. Ostrovskii [12], who obtained sharp bounds for the Valiron deficiencies of entire functions having negative zeros 
and nonintegral order. Taking into account the information provided by (2.13), the results of Ostrovskii imply

If $f(z)$ is an arbitrary entire function of finite order $\lambda$, having only negative zeros, then

$$
\liminf _{r \rightarrow+\infty} \frac{N(r, 0)}{T(r)} \leqq\left\{\begin{array}{lr}
\frac{|\sin \pi \lambda|}{q+|\sin \pi \lambda|} & \left(q \leqq \lambda<q+\frac{1}{2}\right) \\
\frac{|\sin \pi \lambda|}{q+1} & \left(q+\frac{1}{2} \leqq \lambda<q+1\right)
\end{array}\right.
$$

where $q$ is the greatest integer in $\lambda$.

The results of this note may be extended to functions having more general distributions of zeros and poles than those indicated above. For example, (2.14) implies the following information on the deficiency of the zeros of a particularly natural class of entire functions.

COROLlaRY 2.1. If $f(z)$ is an entire function with real zeros and finite order $\lambda$, then

$$
\liminf _{r \rightarrow+\infty} \frac{N(r, 0)}{T(r)} \leqq\left\{\begin{array}{lr}
\frac{|\sin (\pi \lambda / 2)|}{q+|\sin (\pi \lambda / 2)|} & (2 q \leqq \lambda<2 q+1) \\
\frac{|\sin (\pi \lambda / 2)|}{q+1} & (2 q+1 \leqq \lambda<2 q+2) .
\end{array}\right.
$$

These bounds are sharp.

If $f(z)$ is a meromorphic function of finite order, it is always possible in Nevanlinna's deficiency relation

$$
\underset{c}{\sum} \delta(c, f) \leqq 2
$$

to replace one of the $\delta$ 's by the corresponding Valiron deficiency. That is, for functions of finite order (2.16) may be strengthened to

$$
\Delta\left(c_{1}, f\right)+\sum_{c \neq c_{1}} \delta(c, f) \leqq 2,
$$

where $c_{1}$ is an arbitrary complex value, finite or infinite.

To see this, consider the classical estimate

$$
\sum_{v=1}^{q} m\left(r, c_{v}\right) \leqq 2 T(r)+O(\log r) \quad(r \rightarrow+\infty)
$$

(cf. $[11$, p. 256$])$, where the $c_{v}$ are any $q(\geqq 3)$ distinct complex values. Noticing that (2.18) implies 


$$
\underset{r \rightarrow+\infty}{\lim \sup } \frac{m\left(r, c_{1}\right)}{T(r)}+\sum_{v=2}^{q}\left\{\liminf _{r \rightarrow+\infty} \frac{m\left(r, c_{v}\right)}{T(r)}\right\} \leqq 2,
$$

it follows from the definitions (1.1) and (1.2), together with Nevanlinna's first fundamental theorem, that

$$
\Delta\left(c_{1}, f\right)+\sum_{v=2}^{q} \delta\left(c_{v}, f\right) \leqq 2
$$

Since $q$ is arbitrary, (2.19) implies (2.17).

Using this observation with Theorem 2 , it is possible to obtain information on all the deficient values of certain entire functions. In fact, if we choose $c_{1}=0$ and $c_{2}=\infty$ in (2.19), then (2.13) implies

COROLlaRY 2.2. Let $f(z)$ be an entire function with only negative zeros. If either $\mu$ or $\lambda$ is a positive integer,

$$
\delta(c, f)=0
$$

for all values $c \neq 0, \infty$.

Corollary 2.2 is not new, since it is a special case of a result of Edrei and Fuchs [5, Theorem 2]. However, my deduction of this result may be of some interest because of its simplicity.

For orders greater than 1, Theorem 1 may be extended also to meromorphic functions. The following result, stated without proof, follows readily from the methods of this note.

THEOREM 3. Let $f(z)$ be a meromorphic function of order $\lambda$ with negative zeros and positive poles. If $4 \leqq \lambda<+\infty$, then

$$
X^{2}+Y^{2}+2 X Y \cos \left(\frac{2 \pi}{\lambda}\right) \leqq \sin ^{2}\left(\frac{2 \pi}{\lambda}\right),
$$

where $X$ and $Y$ are defined by (2.2).

Although there is no reason to believe that this result is best possible, it is not hard to see that the bound

$$
X+Y<\frac{A}{\lambda} \quad(A=\text { absolute constant }<10)
$$

implied by (2.20) has the correct order of magnitude for large $\lambda$.

In [13] Valiron considers "oriented functions," that is, entire functions whose zeros have arguments tending to a limit; it is possible to suppose, with no loss of generality, that this limit is $\pi$. Valiron's terminology may be extended by defining oriented functions to be those meromorphic functions having zeros $\left\{a_{n}\right\}$ and poles $\left\{b_{n}\right\}$ such that 


$$
\lim _{n \rightarrow+\infty} \arg a_{n}=\pi, \quad \lim _{n \rightarrow+\infty} \arg b_{n}=0 .
$$

Results such as Gol'dberg's lemma [8, p. 108] suggest that functions with negative zeros and positive poles have an extremal character, and provide some justification for the above extension of Valiron's definition to meromorphic functions.

Many of the results of this note may be generalized to oriented functions. I shall not consider this question here because the arguments involved are rather lengthy; it might however be interesting to point out that they are not as straightforward as one might anticipate.

Suppose now that $f(z)$ is a given nonrational meromorphic function, and let $h(z)(\not \equiv 0)$ denote any meromorphic function such that

$$
T(r, h)=o(T(r, f)) \quad(r \rightarrow+\infty) .
$$

Then, by elementary inequalities of Nevanlinna's theory,

$$
T(r, h f) \sim T(r, f)
$$

and

$$
\frac{N(r, h f)}{T(r, h f)}=\frac{N(r, f)}{T(r, f)}+o(1), \frac{N(r, 1 / h f)}{T(r, h f)}=\frac{N(r, 1 / f)}{T(r, f)}+o(1) \quad(r \rightarrow+\infty)
$$

These relations show that the results of this note concerned with the behavior of $\Delta(0, f), \delta(0, f), \Delta(\infty, f), \delta(\infty, f)$ remain true even if infinitely many zeros and poles have unknown arguments, but are sufficiently rare.

In particular, it is clear from (2.21) and (2.22) that we may assume

$$
f(0) \neq 0, \infty \text { and even } f(0)=1
$$

without restricting the generality of our statements.

Finally, to show that nontrivial upper bounds for Valiron deficiences do not in general exist, I prove in $\S 12$ that, with each number $\lambda(0 \leqq \lambda \leqq+\infty)$, it is possible to associate an entire function of order $\lambda$ having negative zeros and such that

$$
\liminf _{r \rightarrow+\infty} \frac{N(r, 0)}{T(r)}=0
$$

Thus, for these functions, $\Delta(0)=\Delta(\infty)=1$.

3. Lemmas on the growth of a real function. Let $G(t)$ be a real, continuous, nondecreasing, unbounded function defined for $t \geqq t_{0}>0$, and denote by

and

$$
\lambda=\limsup _{t \rightarrow+\infty} \frac{\log G(t)}{\log t}
$$

$$
\mu=\liminf _{t \rightarrow+\infty} \frac{\log G(t)}{\log t}
$$


the order and lower order of $G(t)$. In order to describe the growth of $G(t)$ in a manner suitable for applications, it is convenient to introduce the following

Definition. An increasing positive sequence

$$
r_{1}, r_{2}, \cdots, r_{m}, \cdots
$$

is said to be a sequence of Pólya peaks of the second kind, of order $\rho$ $(0 \leqq \rho<+\infty)$, if it is possible to find a pair of associated sequences $\left\{s_{m}\right\},\left\{S_{m}\right\}$ such that

$$
s_{m} \rightarrow+\infty, \quad \frac{r_{m}}{s_{m}} \rightarrow+\infty, \quad \frac{S_{m}}{r_{m}} \rightarrow+\infty
$$

and such that

$$
G(t) \geqq(1+o(1)) \frac{G\left(r_{m}\right)}{r_{m}^{\rho}} t^{\rho} \quad\left(m \rightarrow+\infty, s_{m} \leqq t \leqq S_{m}\right) .
$$

The terminology "Pólya peaks of the second kind" seems appropriate because our sequences (3.1) have the same character as the Pólya peaks defined by Edrei in [2], differing only in that the inequalities (3.3) run in the opposite sense.

That Pólya peaks of the second kind always exist, for functions of finite lower order, is shown by

LEMMA 1. Let $G(t)$ be a real, continuous, nondecreasing and unbounded function defined for $t \geqq t_{0}>0$, and having finitelower order $\mu$.

Then, corresponding to each finite number $\rho$ such that

$$
\mu \leqq \rho \leqq \lambda,
$$

there exists a sequence $\left\{r_{m}\right\}$ of Pólya peaks of the second kind, of order $\rho$.

The proof of Lemma 1 depends upon the following analogue of a result of Edrei [1, Lemma 1] on functions of irregular growth.

LEMMA 2. Let $G(t)$ be a positive, continuous, nondecreasing function defined for $t \geqq t_{0}>0$.

Assume that the order $\lambda$ and the lower order $\mu$ of $G(t)$ satisfy

$$
\mu<\lambda
$$$$
(\lambda \leqq+\infty)
$$

and let $\sigma, \tau$ be given numbers such that

$$
\mu<\sigma<\tau<\lambda .
$$

Then there exist arbitrarily large values of $r$ such that

$$
\frac{G(r)}{r^{\sigma}} \leqq \frac{G(t)}{t^{\sigma}} \quad\left(t_{0} \leqq t \leqq r^{\tau / \sigma}\right) .
$$


Proof of Lemma 2. Let $B(>1)$ be given. Since $\sigma>\mu$, the definition of lower order implies

$$
\liminf _{t \rightarrow+\infty} \frac{G(t)}{t^{\sigma}}=0
$$

and hence there exists $r_{1}>B$ and such that, simultaneously,

$$
\frac{G\left(r_{1}\right)}{r_{1}^{\sigma}} \leqq \frac{G(t)}{t^{\sigma}} \quad\left(t_{0} \leqq t \leqq r_{1}\right)
$$

and

$$
1>\frac{G\left(r_{1}\right)}{r_{1}^{\sigma}} .
$$

Similarly, the inequality $\tau<\lambda$ implies that we may choose $r_{2}>r_{1}$ so that

$$
\frac{G\left(r_{2}\right)}{r_{2}^{\tau}}>1
$$

Using the continuity of $G(t) / t^{\sigma}$, choose $r$ in the interval

$$
r_{1} \leqq r \leqq r_{2}
$$

so that

$$
\frac{G(r)}{r^{\sigma}}=\inf _{r_{1} \leqq t_{12}} \frac{G(t)}{t^{\sigma}} .
$$

Combining (3.7) with (3.11) we find

$$
\frac{G(r)}{r^{\sigma}} \leqq \frac{G(t)}{t^{\sigma}} \quad\left(t_{0} \leqq t \leqq r_{2}\right) .
$$

When

$$
r_{2} \leqq t \leqq r_{2}^{\tau / \sigma},
$$

then $t^{\sigma} \leqq r_{2}^{\tau}$ and we obtain, in view of (3.9), (3.8), (3.11) and the assumption that $G(t)$ is not decreasing,

$$
\frac{G(t)}{t^{\sigma}} \geqq \frac{G\left(r_{2}\right)}{r_{2}^{\tau}}>1>\frac{G\left(r_{1}\right)}{r_{1}^{\sigma}} \geqq \frac{G(r)}{r^{\sigma}} \quad\left(r_{2} \leqq t \leqq r_{2}^{\tau / \sigma}\right) .
$$

Lemma 2 is now an obvious consequence of (3.10), (3.12) and (3.13), and the fact that $B$ is arbitrarily large.

Proof of Lemma 1. We shall define sequences $\left\{r_{m}\right\}$ of Pólya peaks of the second kind such that

$$
r_{m}>m^{m} \quad\left(m \geqq m_{0}\right) .
$$

If we then set 


$$
s_{m}=(1 / m) r_{m}, \quad S_{m}=m r_{m},
$$

it is obvious that the conditions (3.2) will be satisfied, and also that

$$
\left(\frac{t}{r_{m}}\right)^{1 / m}=1+o(1) \quad\left(m \rightarrow+\infty, s_{m} \leqq t \leqq S_{m}\right) .
$$

Assume first that $\mu<\lambda$. For $\rho$ such that

$$
\mu<\rho<\lambda,
$$

choose numbers $\sigma$ and $\tau$ to satisfy

$$
\sigma=\rho<\tau<\lambda,
$$

and use Lemma 2 to find, for each positive integer $m$, a number $r=r_{m}$ satisfying (3.6) and the additional condition (3.14). The inequality (3.3) then follows from the fact that we have chosen $\sigma=\rho$.

When $\rho$ satisfies

$$
\mu=\rho<\lambda,
$$

choose

For

$$
\sigma=\sigma_{m}=\rho+\frac{1}{m}, \quad \tau=\tau_{m}=\rho+\frac{2}{m} .
$$

$$
\mu<\rho=\lambda,
$$

take

$$
\sigma=\sigma_{m}=\rho-\frac{2}{m}, \quad \tau=\tau_{m}=\rho-\frac{1}{m} .
$$

Clearly, in both the cases (3.16) and (3.17)

$$
\mu<\sigma_{m}<\tau_{m}<\lambda \quad\left(m \geqq m_{0}\right)
$$

if $m_{0}$ is large enough, and we may use Lemma 2 to find $r_{m}$ satisfying both (3.6) and (3.14). In view of (3.15), it is obvious that the sequence $\left\{r_{m}\right\}$ thus defined is a sequence of Pólya peaks of the second kind, of order $\rho$.

If $G(t)$ has regular growth, that is, if

then we set

$$
\mu=\rho=\lambda,
$$

$$
\varepsilon=\frac{1}{m}
$$

$$
\begin{aligned}
\phi(t) & =\frac{t^{\rho+\varepsilon}}{G(t)} \\
\psi(t) & =t^{2 \varepsilon}
\end{aligned}
$$


and use an important lemma of Pólya $\left[4\right.$, p. 237] to determine $r_{m}\left(>m^{m}\right)$ so that

$$
\frac{t^{\rho+\varepsilon}}{G(t)} \leqq \frac{r_{m}^{\rho+\varepsilon}}{G\left(r_{m}\right)} \quad\left(t_{0} \leqq t \leqq r_{m}\right)
$$

and

$$
\frac{t^{\rho-\varepsilon}}{G(t)} \leqq \frac{r_{m}^{\rho-\varepsilon}}{G\left(r_{m}\right)}
$$

The verification that the sequence $\left\{r_{m}\right\}$ has all the properties of Pólya peaks of the second kind is now a straightforward consequence of (3.19) and (3.20), together with (3.15) and (3.18).

This completes the proof of Lemma 1.

4. Integrals connected with Weierstrass products of finite genus. Let $E(u, q)$ be the primary factor of genus $q$ :

$$
\begin{aligned}
& E(u, q)=(1-u) \exp \left(u+\frac{1}{2} u^{2}+\cdots+\frac{1}{q} u^{q}\right) \quad(q>0), \\
& E(u, 0)=1-u .
\end{aligned}
$$

Following Valiron, we start from the elementary formula

$$
\log E\left(-\frac{z}{a}, q\right)=(-1)^{q} \int_{a}^{+\infty} \frac{z^{q+1}}{t^{q+1}(t+z)} d t \quad(a>0,|\arg z|<\pi),
$$

and notice that

$$
\int_{0}^{\beta} \log \left|E\left(-\frac{r e^{i \theta}}{a}, q\right)\right| d \theta=(-1)^{q+1} \operatorname{Re}\left\{i \int_{\Gamma} z^{q} d z \int_{a}^{+\infty} \frac{d t}{t^{q+1}(t+z)}\right\},
$$

where $\Gamma$ is the arc of $|z|=r$ defined by

$$
\Gamma=\left\{r e^{i \theta}: 0 \leqq \theta \leqq \beta, 0<\beta<\pi\right\} .
$$

Consider now the Weierstrass product of genus $q$ :

$$
g(z)=\prod_{v=1}^{\infty} E\left(\frac{z}{a_{v}}, q\right),
$$

where all the $a$ 's are negative, and let $n(t, 0)$ be the counting function of its zeros. After interchanging the order of the integrations in the right-hand side of (4.1), an obvious summation leads to

$$
\frac{1}{\pi} \int_{0}^{\beta} \log \left|g\left(r e^{i \theta}\right)\right| d \theta=\frac{(-1)^{q+1}}{\pi} \operatorname{Re}\left\{i \int_{0}^{+\infty} \frac{n(t, 0)}{t^{q+1}} d t \int_{\Gamma} \frac{z^{q}}{t+z} d z\right\},
$$

where the right-hand side may be transformed by an integration by parts. Putting 


$$
J(t)=q \int_{\Gamma} \frac{z^{q}}{t+z} d z+t \int_{\Gamma} \frac{z^{q}}{(t+z)^{2}} d z,
$$

we thus deduce

$$
\frac{1}{\pi} \int_{0}^{\beta} \log \left|g\left(r e^{i \theta}\right)\right| d \theta=\frac{(-1)^{q+1}}{\pi} \operatorname{Re}\left\{i \int_{0}^{+\infty} \frac{N(t, 0)}{t^{q+1}} J(t) d t\right\} .
$$

The form of $J(t)$ may be simplified by setting $z_{1}=r e^{i \beta}$ and integrating by parts the second integral in (4.2); this yields

$$
J(t)=\frac{z_{1}^{q+1}}{t+z_{1}}-\frac{r^{q+1}}{t+r}
$$

and leads to the introduction of

$$
\begin{aligned}
K_{q}(t, r, \beta) & =\frac{(-1)^{q}}{\pi t^{q+1}} \operatorname{Im} J(t) \\
& =\frac{(-1)^{q}}{\pi}\left(\frac{r}{t}\right)^{q+1} \frac{r \sin q \beta+t \sin (q+1) \beta}{t^{2}+2 t r \cos \beta+r^{2}}(q \geqq 0,-\pi<\beta<\pi) .
\end{aligned}
$$

It will be noticed that $K_{q}$ reduces to the Poisson kernel, for the half-plane $\operatorname{Im} z>0$, when $q=0$.

The properties of the kernel $K_{q}$ which we shall require for our proofs are summarized in the following lemma.

LEMMA 3. Let $g(z)$ be a Weierstrass product of genus $q$, having only negative zeros. Then

$$
\begin{aligned}
& \frac{1}{\pi} \int_{0}^{\beta} \log \left|g\left(r e^{i \theta}\right)\right| d \theta=\int_{0}^{\infty} N(t, 0) K_{q}(t, r, \beta) d t \quad(-\pi<\beta<\pi), \\
& \lim _{\beta \rightarrow \pi-} \int_{0}^{\infty} N(t, 0) K_{q}(t, r, \beta) d t=N(r, 0), \\
& (-1)^{q} K_{q}(t, r, \theta) \geqq 0 \text { when } 0 \leqq \theta \leqq \frac{\pi}{q+1}, \\
& \int_{0}^{\infty} s^{\rho} K_{q}(s, 1, \beta) d s=\frac{\sin \beta \rho}{\sin \pi \rho} \quad(q<\rho<q+1,-\pi<\beta<\pi) .
\end{aligned}
$$

Proof. The representation (4.5) is a consequence of (4.3) and (4.4) when $0 \leqq \beta<\pi$. Its validity when $-\pi<\beta<0$ then follows from the observation that $\log \left|g\left(r e^{i \theta}\right)\right|$ is an even function of $\theta$ since $g(z)$ is real for real $z$, and thus both sides of (4.5) are odd functions of $\beta$ defined for the whole interval $-\pi<\beta<\pi$.

By (4.5) and Jensen's theorem, 


$$
\begin{aligned}
\int_{0}^{\infty} N(t, 0) K_{q}(t, r, \beta) d t & =\frac{1}{\pi} \int_{0}^{\beta} \log \left|g\left(r e^{i \theta}\right)\right| d \theta \\
& =N(r, 0)-\frac{1}{\pi} \int_{\beta}^{\pi} \log \left|g\left(r e^{i \theta}\right)\right| d \theta
\end{aligned}
$$

and hence (4.6) follows by letting $\beta \rightarrow \pi-$.

The inequality (4.7) is an immediate consequence of the definition (4.4).

An elementary contour integration yields the formula

(4.9) $\frac{1}{\pi} \int_{0}^{\infty} s^{\alpha} \frac{\sin \beta}{s^{2}+2 s \cos \beta+1} d s=\frac{\sin \beta \alpha}{\sin \pi \alpha}(-1<\alpha<1, \alpha \neq 0 ;-\pi<\beta<\pi)$.

Using (4.4), and assuming $\beta \neq 0$ because (4.8) is trivial otherwise, (4.9) implies

$$
\begin{aligned}
\int_{0}^{\infty} s^{\rho} K_{q}(s, 1, \beta) d s & =\frac{(-1)^{q}}{\pi}\left\{\sin q \beta \int_{0}^{\infty} \frac{s^{\rho-q-1}}{s+2 s \cos \beta+1} d s\right. \\
& \left.\quad+\sin (q+1) \beta \int_{0}^{\infty} \frac{s^{\rho-q}}{s^{2}+2 s \cos \beta+1} d s\right\} \\
& =(-1)^{q}\left\{\frac{\sin q \beta}{\sin \beta} \frac{\sin (\rho-q-1) \beta}{\sin (\rho-q-1) \pi}+\frac{\sin (q+1) \beta}{\sin \beta} \frac{\sin (\rho-q) \beta}{\sin (\rho-q) \pi}\right\} \\
& =\frac{\sin \beta \rho}{\sin \pi \rho},
\end{aligned}
$$

which establishes (4.8) and completes the proof of Lemma 3.

5. A lower bound for the logarithmic mean $m(r, f)$. Let $f(z)$ be an arbitrary function of finite order $\lambda$ whose zeros and poles of positive modulus are denoted by $\left\{a_{n}\right\}$ and $\left\{b_{n}\right\}$, respectively, and let $q$ be the smallest non-negative integer such that

$$
\Sigma \frac{1}{\left|a_{n}\right|^{q+1}}+\Sigma \frac{1}{\left|b_{n}\right|^{q+1}}<+\infty .
$$

Then it is well known that $f(z)$ may be expressed in the form

$$
f(z)=z^{m} e^{Q(z)} \frac{\prod E\left(\frac{z}{a_{n}}, q\right)}{\prod E\left(\frac{z}{b_{n}}, q\right)},
$$

where $Q(z)$ is a polynomial of degree $d \leqq \lambda$, and $m$ is an integer.

If all the $a_{n}$ are negative and all the $b_{n}$ are positive, we may apply the results of Lemma 3 to $g(z)=f(z) z^{-m} e^{-Q(z)}$ to obtain 


$$
\begin{aligned}
& \frac{1}{\pi} \int_{0}^{\beta} \log \left|g\left(r e^{i \theta}\right)\right| d \theta+N(r, \infty) \\
& \quad=\int_{0}^{\infty} N(t, 0) K_{q}(t, r, \beta) d t+\int_{0}^{\infty} N(t, \infty) K_{q}(t, r, \pi-\beta) d t \quad(0<\beta<\pi) .
\end{aligned}
$$

As we observed in $\S 4,\left|g\left(r e^{i \theta}\right)\right|$ is an even function of $\theta$ and hence the definition of $m(r, g)$ implies

$$
m(r, g) \geqq \frac{1}{\pi} \int_{\delta} \log \left|g\left(r e^{i \theta}\right)\right| d \theta,
$$

where $\mathscr{E}$ is any measurable subset of $0 \leqq \theta \leqq \pi$.

This leads to the following inequality, basic for my proofs:

$$
m(r, f) \geqq \sum_{v=1}^{l} \int_{0}^{\infty} N(t, 0)\left[K_{q}\left(t, r, \beta_{v}\right)-K_{q}\left(t, r, b_{v}\right)\right] d t
$$

$$
\begin{array}{r}
+\sum_{v=1}^{l} \int_{0}^{\infty} N(t, \infty)\left[K_{q}\left(t, r, \pi-\beta_{v}\right)-K_{q}\left(t, r, \pi-b_{v}\right)\right] d t-A\left(r^{d}+\log r\right) \\
\left(0<b_{1}<\beta_{1}<\cdots<b_{l}<\beta_{l}<\pi, r \geqq 1\right) ;
\end{array}
$$

it is a consequence of (5.3), (5.4) and the obvious relation

$$
m(r, g) \leqq m(r, f)+A\left(r^{d}+\log r\right)
$$

valid for a suitable constant $A$. The usefulness of (5.5) is due to the fact that the intervals $\left(b_{v}, \beta_{v}\right)$ are abirtrary disjoint subintervals of $(0, \pi)$.

It will be useful to consider one aspect of the relative rates of growth of the various factors which appear in the decomposition (5.2) of a meromorphic function $f(z)$ of finite order.

If $\pi(z)$ is an arbitrary convergent Weierstrass product $\left({ }^{2}\right)$ of genus $q$, then a classical estimate $[8$, p. 29$]$ leads immediately to

$$
T(r, \pi(z))=o\left(r^{q+1}\right) \quad(r \rightarrow+\infty) .
$$

Thus (5.6) and Nevanlinna's first fundamental theorem imply that the quotient of Weierstrass products

$$
g(z)=\frac{\prod E\left(\frac{z}{a_{n}}, q\right)}{\prod E\left(\frac{z}{b_{n}}, q\right)}=\frac{\pi(z)}{\pi_{2}(z)}
$$

satisfies

(2) I do not say canonical because the estimate (5.6) is true even if $q$ is larger than its canonical choice. 


$$
T(r, g) \leqq T\left(r, \pi_{1}\right)+T\left(r, \pi_{2}\right)=o\left(r^{q+1}\right) \quad(r \rightarrow+\infty) .
$$

It follows trivially that the characteristic of

$$
h(z)=1 /\left(z^{m} g(z)\right)
$$

also satisfies

$$
T(r, h)=o\left(r^{q+1}\right) \quad(r \rightarrow+\infty) .
$$

In view of the definitions (5.2) and (5.8),

$$
h(z) f(z)=e^{Q(z)}
$$

and hence for a suitable constant $\alpha(\geqq 0)$

$$
T(r, f) \geqq T\left(r, e^{Q}\right)-T(r, h)=(\alpha+o(1)) r^{d}-o\left(r^{q+1}\right) \quad(r \rightarrow+\infty) .
$$

Suppose now that $d \geqq q+1$. Then (5.9) and (5.10) imply

$$
T(r, h)=o(T(r, f)) \quad(r \rightarrow+\infty),
$$

so that we may use the relations (2.21), (2.22) to deduce

$$
T(r, f) \sim T\left(r, e^{Q}\right) \sim \alpha r^{d} \quad(0<\alpha=\text { const. })
$$

and

$$
\frac{N(r, f)}{T(r, f)}=\frac{N\left(r, e^{Q}\right)}{T\left(r, e^{Q}\right)}+o(1)=o(1), \quad \frac{N(r, 1 / f)}{T(r, f)}=o(1) \quad(r \rightarrow+\infty) .
$$

The asymptotic relations (5.11) and (5.12) show that the type of behavior we are investigating in this note is completely determined when $d>q$, and hence there will be no loss of generality if, in the sequel, we always assume $d \leqq q$.

6. Proof of Theorem 1 when $\lambda<1$. Starting from the inequality (5.5), we notice that necessarily $q=d=0$ when $\lambda<1$. If we take $l=1$ in (5.5), and let $b_{1} \rightarrow 0+$, then (4.6) implies

$$
\begin{aligned}
T(r) & =m(r, f)+N(r, \infty) \\
& \geqq \int_{0}^{\infty} N(t, 0) K_{0}(t, r, \beta) d t+\int_{0}^{\infty} N(t, \infty) K_{0}(t, r, \pi-\beta) d t-A \log r,
\end{aligned}
$$

valid for all $\beta=\beta_{1}$ in $0<\beta<\pi$ and for all $r>0$.

If $X=1-\Delta(0, f)>0$, choose $\bar{X}$ so that $0<\bar{X}<X$; if $X=0$, let $\bar{X}=0$. Hence, in any case,

$$
N(t, 0) \geqq X T(t)
$$

for all sufficiently large $t$. Similarly, we have 


$$
N(t, \infty) \geqq \bar{P} T(t)
$$

for all large $t$, where $\bar{Y}$ is any number in

$$
0<\bar{Y}<Y=1-\Delta(\infty, f)
$$

if $Y>0$, and $Y=0$ if $Y=0$.

The condition (2.4) shows that (2.3) is trivial when $\lambda=0$, and thus we may as well assume that $0<\lambda<1$.

Choose any positive number $\rho$ such that $\mu \leqq \rho \leqq \lambda$, and let $\left\{r_{m}\right\}$ be a sequence of Pólya peaks of the second kind, of order $\rho$, for the function $T(t)$. In view of the positivity of $K_{0}(t, r, \beta)$ and the inequalities (6.1), (6.2), (6.3), we obtain the relation

$$
T\left(r_{m}\right) \geqq X T\left(r_{m}\right)(1+o(1)) \int_{s}^{s}\left(t / r_{m}\right)^{\rho} K_{0}\left(t, r_{m}, \beta\right) d t
$$

$$
+\bar{Y} T\left(r_{m}\right)(1+o(1)) \int_{s}^{s}\left(t / r_{m}\right)^{\rho} K_{0}\left(t, r_{m}, \pi-\beta\right) d t-A \log r \quad(m \rightarrow \infty) .
$$

(Here we have used implicitly the fact that $s_{m} \rightarrow+\infty$; this consequence of (3.2) simplifies the manipulation of asymptotic inequalities such as (6.2) and (6.3).)

Making the change of variable $s=t / r_{m}$, and dividing by $T\left(r_{m}\right),(6.4)$ may be rewritten as

(6.5) $1+o(1) \geqq X \int_{s_{m} / r . .}^{S_{n} / r} s^{\rho} K_{0}(s, 1, \beta) d s+\bar{Y} \int_{s_{m} / r_{m} . .}^{S_{m} / r_{m}} s^{\rho} K_{0}(s, 1, \pi-\beta) d s(m \rightarrow \infty)$.

The properties (3.2) of Pólya peaks of the second kind thus lead to

$$
1 \geqq \not{X} \int_{0}^{\infty} s^{\rho} K_{0}(s, 1, \beta) d s+\bar{Y} \int_{0}^{\infty} s^{\rho} K_{0}(s, 1, \pi-\beta) d s .
$$

Evaluating these integrals explicitly by means of (4.8), and letting $X \rightarrow X, \bar{Y} \rightarrow Y$, we obtain

$$
\sin \pi \rho \geqq X \sin \beta \rho+Y \sin (\pi-\beta) \rho \quad(\mu \leqq \rho \leqq \lambda) .
$$

We have established (6.6) for any $\beta$ in $0<\beta<\pi$, but since the right-hand side is continuous (6.6) continues to hold when $\beta=0$ or $\beta=\pi$.

In order to obtain the strongest possible bounds on the quantities $X$ and $Y$, set

$$
\rho=\lambda
$$

and

$$
\beta=\frac{1}{\lambda} \tan ^{-1}\left(\frac{X-Y \cos \pi \lambda}{Y \sin \pi \lambda}\right),
$$

the branch of the inverse tangent being that one for which $\tan ^{-1}(0)=0$. 
We must verify that this choice of $\beta$ implies

$$
0 \leqq \beta \leqq \pi,
$$

since otherwise its use in (6.6) would be meaningless. This is obvious when $1 / 2 \leqq \lambda \leqq 1$, since then (6.8) implies

$$
0 \leqq \beta \leqq \frac{\pi}{2 \lambda} .
$$

For orders $\lambda$ in the interval $0<\lambda<1 / 2$, the hypothesis (2.4) leads to

$$
0 \leqq \frac{X-Y \cos \pi \lambda}{Y \sin \pi \lambda}=\frac{X \sin ^{2} \pi \lambda-\cos \pi \lambda(Y-X \cos \pi \lambda)}{Y \sin \pi \lambda} \leqq \frac{\sin \pi \lambda}{\cos \pi \lambda},
$$

and hence to

$$
0 \leqq \tan ^{-1}\left(\frac{X-Y \cos \pi \lambda}{Y \sin \pi \lambda}\right) \leqq \pi \lambda .
$$

In view of the definition (6.8), these inequalities imply (6.9).

From (6.6) we deduce

$$
\sin ^{2} \pi \lambda \geqq\{(X-Y \cos \pi \lambda) \sin \beta \lambda+Y \sin \pi \lambda \cos \beta \lambda\}^{2},
$$

and from (6.8)

$$
0=\{(X-Y \cos \pi \lambda) \cos \beta \lambda-Y \sin \pi \lambda \sin \beta \lambda\}^{2},
$$

each side of which we may add to the corresponding side of (6.10). After some obvious reductions we obtain

$$
X^{2}+Y^{2}-2 X Y \cos \pi \lambda \leqq \sin ^{2} \pi \lambda
$$

which proves Theorem 1 for orders $\lambda<1$.

7. Proof of Theorem 1 when $f(z)$ has order 1 and genus 0 . The definition of genus implies $q=0$ and $d=0$, and by assumption $\lambda=1$. Then $f(z)$ has the representation (5.2) and the inequality (6.5) is still valid, with $\rho=1$ and, say, $\beta=\pi / 2$.

Recalling the definition (4.4) of $K_{0}(s, 1, \pi / 2)$ and the property (3.2) of Pólya peaks of the second kind, it is clear that as $m \rightarrow \infty$ the integrals in (6.5) e?ch tend to $+\infty$. Thus we have necessarily $X=Y=0$; but this implies $X=Y=0$, which is the assertion of Theorem 1 when $\lambda=1$.

8. Proof of Theorem 1 when $f(\mathrm{z})$ has genus 1 . A function $f(z)$ of genus 1 can always be represented in the form (5.2), where $q=0$ and $d=1$ or $q=1$ and $d \leqq 1$.

We may dismiss at once the case characterized by $q=0$ and $d=1$, as the discussion at the end of $\$ 5$ explains, and assume from this point on that $q=1$. 
Thus the proof of Theorem 1 will be complete if we can establish that

$$
X=Y=0
$$

when $\lambda=q=1$. This information is contained in the following extension of Theorem 1, which we prove in order to indicate how the methods of this note may be used to obtain bounds on the deficiencies of meromorphic functions of orders greater than 1.

THEOREM 1a. Let $f(z)$ be a meromorphic function of order $\lambda(1 \leqq \lambda<2)$ and lower order $\mu$.

If $f(z)$ has negative zeros $a_{n}$ and positive poles $b_{n}$ such that

$$
\Sigma \frac{1}{\left|a_{n}\right|}+\Sigma \frac{1}{b_{n}}=+\infty
$$

then necessarily

$$
X+Y \leqq \liminf _{r \rightarrow+\infty} \frac{N(r, 0)+N(r, \infty)}{T(r)} \leqq \inf _{\mu \leqq \rho \leqq \lambda} \frac{|\sin \pi \rho|}{\sin \left(\frac{\pi \rho}{2}\right)+|\sin \pi \rho|}
$$

where $X$ and $Y$ are the quantities defined by (2.2).

If $\lambda=q=1,(8.2)$ is satisfied. Hence (8.3) yields $X+Y=0$, which implies (8.1).

To establish Theorem 1a, we return to the inequality (5.5) and choose $l=1$, $b_{1}=\pi / 2$. Letting $\beta_{1} \rightarrow \pi-$, we thus obtain

$$
T(r) \geqq N(r, 0)+N(r, \infty)-\int_{0}^{\infty}[N(t, 0)+N(t, \infty)] K_{1}\left(t, r, \frac{\pi}{2}\right) d t-A r
$$

For convenience, set

$$
N(t)=N(t, 0)+N(t, \infty) \text {. }
$$

Using the definition (4.4) of $K_{1}(t, r, \pi / 2),(8.4)$ becomes

$$
T(r) \geqq N(r)+\frac{r}{\pi} \int_{0}^{\infty} \frac{N(t)}{t^{2}} \frac{r^{2}}{t^{2}+r^{2}} d t-A r,
$$

and hence

$$
\frac{T(r)}{r} \geqq \frac{1}{2 \pi} \int_{0}^{r} \frac{N(t)}{t^{2}} d t-A
$$

In view of the condition (8.2), the integral in (8.7) tends to $+\infty$ with $r$ (cf. for instance $[8$, p. 25]), so that

$$
\lim _{r \rightarrow \infty} \frac{T(r)}{r}=+\infty
$$


In particular, the lower order $\mu$ of $f(z)$ satisfies

$$
\mu \geqq 1 \text {. }
$$

Denote by $\mu_{1}$ and $\lambda_{1}$ the lower order and order, respectively, of the function $N(t)$. Then the inequality

$$
N(t) \leqq 2 T(t)+O(1) \quad(t \rightarrow+\infty)
$$

(an obvious consequence of Nevanlinna's first fundamental theorem) shows that $\mu_{1} \leqq \mu$ and $\lambda_{1} \leqq \lambda$. Using another well-known result of Nevanlinna (cf. [10, p. 51] or [8, p. 101]), we find also that $\lambda_{1}=\lambda$ when $\lambda$ is not a positive integer. Combining these observations with (8.9), we see that the quantities $\mu_{1}$ and $\lambda_{1}$ satisfy

$$
\mu_{1} \leqq \mu \text { and } 1 \leqq \mu \leqq \lambda_{1}=\lambda<2
$$

for the functions of Theorem 1a.

Select any number $\rho$ such that $\mu \leqq \rho \leqq \lambda$, and let $\left\{r_{m}\right\}$ be a sequence of Pólya peaks of the second kind, of order $\rho$, for the function $N(t)$. Such a sequence always exists, by virtue of Lemma 1 and the relations (8.10).

Now the inequality (4.7) shows that the kernel $-K_{1}(t, r, \pi / 2)$ is positive; thus we may use (8.4), (8.5) and (3.3) to deduce

$$
T\left(r_{m}\right) \geqq N\left(r_{m}\right)-(1+o(1)) N\left(r_{m}\right) \int_{s . .}^{S}\left(t / r_{m}\right)^{\rho} K_{1}\left(t, r_{m}, \frac{\pi}{2}\right) d t-A r_{m} \quad(m \rightarrow+\infty) .
$$

Making the change of variable $s=t / r_{m}$, dividing by $T\left(r_{m}\right)$, and using (8.8), we obtain

$$
1 \geqq\left[1-\int_{0}^{\infty} s^{\rho} K_{1}\left(s, 1, \frac{\pi}{2}\right) d s\right] \cdot \limsup _{m \rightarrow+\infty} \frac{N\left(r_{m}\right)}{T\left(r_{m}\right)} .
$$

If $\rho>1$, we may use (4.8) to evaluate the integral in (8.11); this leads to

$$
\limsup _{m \rightarrow+\infty} \frac{N\left(r_{m}\right)}{T\left(r_{m}\right)} \leqq \frac{|\sin \pi \rho|}{\sin \left(\frac{\pi \rho}{2}\right)+|\sin \pi \rho|} \quad(1<\rho \leqq \lambda) .
$$

If $\rho=1$, the integral in (8.11) is divergent. But then, in view of the positivity of $-K_{1}(s, 1,(\pi / 2))$, we must have

$$
\limsup _{m \rightarrow+\infty} \frac{N\left(r_{m}\right)}{T\left(r_{m}\right)}=0
$$

in this case, and hence (8.12) remains valid even when $\rho=1$.

This shows that (8.3) is true in general, and completes the proof of Theorem 1a.

9. Proof of Theorem 2. The functions $f(z)$ of Theorem 2 are of the form

$$
f(z)=e^{Q(z)} g(z),
$$


where $Q(z)$ is a polynomial of degree $d$ and $g(z)$ is a canonical product of genus $q$ having only negative zeros. As noted in $\S 5$, we may as well assume that $d \leqq q$. (If $d>q$, then $\mu=\lambda=d$ and hence (2.11) holds in this case.)

By hypothesis, the order $\lambda$ of $f(z)$ is greater than 1 , so that the genus $q$ of $g(z)$ must satisfy

$$
q \geqq 1 \text {. }
$$

A result of Edrei and Fuchs [3, pp. 308, 309] on the growth of entire functions with negative zeros shows that the functions $f(z)$ of Theorem 2 satisfy

$$
\lim _{r \rightarrow \infty} \frac{T(r, f)}{r^{q}}=+\infty
$$

In particular, the lower order $\mu$ of $f(z)$ satisfies

$$
\mu \geqq q \text {. }
$$

As in $\S 8$, denote by $\mu_{1}$ and $\lambda_{1}$ the lower order and order, respectively, of $N(t, 0)$. Using again the arguments of $\S 8$ that led to the inequalities (8.10), we deduce from (9.2) and (5.7) that

$$
\mu_{1} \leqq \mu \text { and } q \leqq \mu \leqq \lambda_{1}=\lambda \leqq q+1 .
$$

This proves the assertions (2.10) and (2.11). It follows from the relations (9.3) and Lemma 1 of $\S 3$ that the function $N(t, 0)$ has Pólya peaks, of the second kind, of all orders $\rho$ such that

$$
\mu \leqq \rho \leqq \lambda .
$$

Let $\rho$ denote a fixed number satisfying (9.4). When $q$ is an odd integer, we return to (5.5) and choose $l=1, b_{1}=\pi / 2 \rho$. Letting $\beta_{1} \rightarrow \pi-,(4.6)$ implies

$$
T(r, f) \geqq N(r, 0)-\int_{0}^{\infty} N(t, 0) K_{q}\left(t, r, \frac{\pi}{2 \rho}\right) d t-A\left(r^{d}+\log r\right) \quad(r \geqq 1) .
$$

When $q$ is even, choose $l=2$ in (5.5) and put $b_{1}=0, \beta_{1}=\pi / 2 \rho$ and $b_{2}=\pi / q$ Letting $\beta_{2} \rightarrow \pi-$, we obtain

$$
\begin{array}{r}
T(r, f) \geqq N(r, 0)+\int_{0}^{\infty} N(t, 0)\left[K_{q}\left(t, r, \frac{\pi}{2 \rho}\right)-K_{q}\left(t, r, \frac{\pi}{q}\right)\right] d t-A\left(r^{d}+\log r\right) \\
(r \geqq 1) .
\end{array}
$$

The definition (4.4) shows that $K_{q}(t, r, \pi / q)$ is negative when $q(>0)$ is even, and hence (9.6), (9.5) and the inequality $d \leqq q$ imply

$$
T(r, f) \geqq N(r, 0)+(-1)^{q} \int_{0}^{\infty} N(t, 0) K_{q}\left(t, r, \frac{\pi}{2 \rho}\right) d t-A r^{q} \quad(r \geqq 1)
$$

for all $q \geqq 1$, odd or even. 
It is important to notice that $(-1)^{q} K_{q}(t, r, \pi / 2 \rho)$ is a positive kernel; this follows at once from (4.7), (9.4) and (9.3).

Since $\rho$ satisfies (9.4), we may choose a sequence $\left\{r_{m}\right\}$ of Pólya peaks of the second kind, of order $\rho$, for $N(t, 0)$. Using (9.7) and the definition of the peaks $r_{m}$, we deduce

$$
T\left(r_{m}, f\right) \geqq N\left(r_{m}, 0\right)+(-1)^{q} \int_{s_{n}}^{S} N(t, 0) K_{q}\left(t, r_{m}, \frac{\pi}{2 \rho}\right) d t-A r_{m}^{q}
$$

$$
\begin{array}{r}
\geqq N\left(r_{m}, 0\right)\left\{1+(1+o(1))(-1)^{q} \int_{s \ldots .}^{S}\left(t / r_{m}\right)^{\rho} K_{q}\left(t, r_{m}, \frac{\pi}{2 \rho}\right) d t\right\}-A r_{m}^{q} \\
(m \rightarrow \infty) .
\end{array}
$$

Dividing by $T\left(r_{m}, f\right)$, and taking into account (9.1), (9.8) implies

$$
\underset{m \rightarrow+\infty}{\lim \sup _{m}}\left\{1+(-1)^{q} \int_{s_{m} / r_{m}}^{S_{m_{m} / r_{m}}} s^{\rho} K_{q}\left(s, 1, \frac{\pi}{2 \rho}\right) d s\right\} \frac{N\left(r_{m}, 0\right)}{T\left(r_{m}, f\right)} \leqq 1 .
$$

If $q<\rho<q+1$, the integral in (9.9) tends to a limit which we may evaluate by means of (4.8). Thus we obtain

$$
\limsup _{m \rightarrow+\infty} \frac{N\left(r_{m}, 0\right)}{T\left(r_{m}, f\right)} \leqq \frac{|\sin \pi \rho|}{1+|\sin \pi \rho|} .
$$

In view of (9.2), $\rho=q$ or $\rho=q+1$ are possible if $f(z)$ has lower order $\mu=q$ of if $\lambda=q+1$. In these cases, the integral in (9.9) tends to $+\infty$ when $m \rightarrow+\infty$, and hence (9.10) remains valid even when $\rho=q$ or $q+1$.

Since (9.10) implies (2.12), the proof of Theorem 2 is complete.

We remark that, by a rather more careful analysis of the set of $\theta$ for which $(-1)^{q} K_{q}(t, r, \theta)$ remains positive as $t$ varies from 0 to $+\infty$, it is possible to deduce

$$
\liminf _{r \rightarrow+\infty} \frac{N(r, 0)}{T(r, f)} \leqq A \min _{\mu \leqq \rho \leqq \lambda} \frac{|\sin \pi \rho|}{q+|\sin \pi \rho|},
$$

where $q$ is the greatest integer in $\rho$ and $A<2$. Further, when

$$
\left|q+\frac{1}{2}-\rho\right| \leqq \frac{1}{2 q}
$$

the constant $A$ may be replaced by 1 , thus making (9.11) a best possible inequality for at least some values of $\lambda$ and $\mu$.

10. Proof of Corollary 1.1. Since the limits (2.6) exist, a result [1, Theorem 3] on the Nevanlinna deficient values of functions of lower order $\mu<1$ implies 


$$
\begin{gathered}
X^{2}+Y^{2}-2 X Y \cos \pi \mu \geqq \sin ^{2} \pi \mu, \\
X=1 \text { if } Y<\cos \pi \mu, \\
Y=1 \text { if } X<\cos \pi \mu .
\end{gathered}
$$

Since (10.1) and (2.3) are incompatible when $\mu<\lambda$ and $\lambda \leqq 1$, in view of the hypothesis

$$
X<1 \text { and } Y<1
$$

we have $\mu=\ell$ and

$$
X^{2}+Y^{2}-2 X Y \cos \pi \lambda=\sin ^{2} \pi \lambda .
$$

To establish (2.8), we refer to a result of Edrei [2, p. 87] on meromorphic functions of lower order $\mu(0<\mu<1)$. This result implies, for the functions of Corollary 1.1, that

$$
\sin \pi \lambda \leqq X \sin \beta \lambda+Y \sin (\pi-\beta) \lambda,
$$

where $\beta$ is any limit point of either of the two sequences

$$
\left\{\text { meas } \varepsilon_{\infty}\left(r_{k}\right)\right\}_{k=1}^{\infty},\left\{\pi \text {-meas } \varepsilon_{0}\left(r_{k}\right)\right\}_{k=1}^{\infty} \text {. }
$$

Here $\left\{r_{k}\right\}$ is an arbitrary sequence of Pólya peaks [2, p. 81] of $T(r, f)$, while $\varepsilon_{0}(r)$ is the set of $\theta$ in $0 \leqq \theta \leqq \pi$ for which $f\left(r e^{i \theta}\right)$ is "close to 0 ," i.e.

$$
\varepsilon_{0}(r)=\left\{\theta:\left|f\left(r e^{i \theta}\right)\right|<\frac{1}{r}, 0 \leqq \theta \leqq \pi\right\}
$$

and $\varepsilon_{\infty}(r)$ is the corresponding set of $\theta$ for which $f\left(r e^{i \theta}\right)$ is "close to $\infty$ ":

$$
\varepsilon_{\infty}(r)=\left\{\theta:\left|f\left(r e^{i \theta}\right)\right|>r, 0 \leqq \theta \leqq \pi\right\} .
$$

Comparing (10.3) with (6.6), and recalling that $\mu=\lambda$, we obtain

$$
\sin \pi \lambda=X \sin \beta \lambda+Y \sin (\pi-\beta) \lambda,
$$

valid for any limit point $\beta$ of either of the sequences (10.4).

Making the substitution

$$
k=\tan \left(\frac{\beta \lambda}{2}\right)
$$

in (10.7), we obtain the quadratic equation

$$
k^{2}(1+Y) \sin \pi \lambda-2 k(X-Y \cos \pi \lambda)+(1-Y) \sin \pi \lambda=0,
$$

which determines $k$. The relation (10.2) expresses the fact that the discriminant of this equation vanishes; hence its two roots are equal and their product is given by 


$$
k^{2}=\frac{1-Y}{1+Y}
$$

from which it follows that

$$
\cos \beta \lambda=\frac{1-k^{2}}{1+k^{2}}=Y
$$

Since $\cos \beta \lambda$ decreases as $\beta$ varies from 0 to $\pi$, the equation (10.8) determines $\beta$ uniquely in the range $0 \leqq \beta \leqq \pi$.

When $0<\lambda \leqq 1 / 2,(10.2)$ and (10.8) imply

so that in fact

$$
\cos \pi \lambda<Y=\cos \beta \lambda<1 \text {, }
$$

$$
0<\beta<\pi
$$

For orders $\lambda$ in $1 / 2<\lambda<1,(10.2)$ implies

$$
0 \leqq Y \leqq \sin \pi \lambda \text {. }
$$

Combining this information with (10.8) yields

$$
\pi-\frac{\pi}{2 \lambda} \leqq \beta \leqq \frac{\pi}{2 \lambda}
$$

which shows that $\beta$ is subject to a more stringent condition than (10.9) when $\lambda>1 / 2$.

The inequalitiies (10.9) and (10.10), together with (10.8), prove (2.8).

The assertion (2.9) follows from the fact that $\beta$ is the limit of each of the two sequences (10.4), together with the observation that $\left|f\left(r e^{i \theta}\right)\right|$ is an even function of $\theta$, which decreases as $\theta$ increases from 0 to $\pi$.

This completes the proof of Corollary 1.1.

11. Proof of Corollary 2.1. Let the entire function

$$
f(z)=z^{m} e^{Q(z)} g(z)
$$

have only real zeros and order $\lambda$. As in $\S 9, g(z)$ denotes a canonical product of genus $q$ and $Q(z)$ has degree $d$.

We assume as usual that $d \leqq q$. Further, the remarks at the end of $\S 2$ show that there is no loss of generality in assuming that $f(0)=1$, so that the exponent $m$ in (11.1) vanishes.

Then the auxiliary function $F(z)$ defined by

$$
F\left(z^{2}\right)=f(z) f(-z)
$$

is an entire function of order $\lambda / 2$, having only positive zeros. Hence we may apply the inequality (2.14) to $F(z)$, to deduce 


$$
\liminf _{r \rightarrow+\infty} \frac{N\left(r, \frac{1}{F}\right)}{T(r, F)} \leqq\left\{\begin{array}{c}
\frac{|\sin (\pi \lambda / 2)|}{p+|\sin (\pi \lambda / 2)|} \\
\frac{|\sin (\pi \lambda / 2)|}{p+1}
\end{array}\right.
$$

$$
\begin{gathered}
\left(p \leqq \frac{\lambda}{2}<p+\frac{1}{2}\right), \\
\left(p+\frac{1}{2} \leqq \frac{\lambda}{2}<p+1\right),
\end{gathered}
$$

where $p$ denotes the greatest integer in $\lambda / 2$.

Since, by the elements of the theory,

$$
N\left(r^{2}, 1 / F(z)\right)=N\left(r, 1 / F\left(z^{2}\right)\right)=2 N(r, 1 / f(z))
$$

and

$$
m\left(r^{2}, F(z)\right)=m\left(r, F\left(z^{2}\right)\right) \leqq 2 m(r, f(z)),
$$

we obtain

$$
\liminf _{r \rightarrow+\infty} \frac{N(r, 1 / f)}{T(r, f)} \leqq \liminf _{r \rightarrow+\infty} \frac{N(r, 1 / F)}{T(r, F)}
$$

Corollary 2.1 now follows on comparing (11.5) with (11.2).

To see that (2.15) is sharp, we observe first that when $\lambda$ is a positive even integer (2.15) implies

$$
\liminf _{r \rightarrow+\infty} \frac{N(r, 0)}{T(r)}=0
$$

and hence equality is always attained in (2.15) for these orders.

In view of a well-known result $[14, p .133]$, the function

$$
f_{0}(z)=\prod_{n=1}^{\infty}\left(1+\frac{z}{e^{n}}\right),
$$

for which

$$
n(r, 0) \sim \log r \quad(r \rightarrow+\infty),
$$

must satisfy

$$
N(r, 0) \sim \log M\left(r, f_{0}\right) \sim \log ^{2} r \quad(r \rightarrow+\infty) .
$$

Thus

$$
N(r, 0) \sim T\left(r, f_{0}\right) \quad(r \rightarrow+\infty),
$$

so that (2.15) cannot be improved when $\lambda=0$.

Assume now that $\lambda(0<\lambda<+\infty)$ is not an even integer, and define

$$
f_{\lambda}(z)=\prod_{n=1}^{\infty} E\left(\frac{z}{-n^{\gamma}}, q_{1}\right) \cdot \prod_{n=1}^{\infty} E\left(\frac{z}{n^{\gamma}}, q_{1}\right) \quad\left(\gamma=\frac{1}{\lambda}, q_{1}=[\lambda]\right) .
$$


Then the function

$$
\left.F_{\sigma}(\zeta)=\prod_{n=1}^{\infty} E\left(\frac{\zeta}{n^{2 \gamma}},\left[q_{1} / 2\right]\right)=F_{\sigma}\left(z^{2}\right)=f_{\lambda}(z) \quad \zeta=R e^{i \phi}=z^{2}\right)
$$

is entire and of order $\sigma=\lambda / 2$ as a function of $\zeta$, and has positive zeros. The formulas of $[10$, pp. 18,19$]$ show that $F_{\sigma}(\zeta)$ satisfies

$$
\frac{N\left(r, \frac{1}{F_{\sigma}(\zeta)}\right)}{T\left(r, F_{\sigma}(\zeta)\right)} \sim\left\{\begin{array}{lr}
\frac{|\sin \pi \sigma|}{q+|\sin \pi \sigma|} & \left(q<\sigma<q+\frac{1}{2}\right), \\
\frac{|\sin \pi \sigma|}{q+1} & \left(q+\frac{1}{2} \leqq \sigma<q+1\right) .
\end{array}\right.
$$

Using the relation

$$
\frac{N\left(r, 1 / f_{\lambda}(z)\right)}{T\left(r, f_{\lambda}(z)\right)}=\frac{N\left(r^{2}, 1 / F_{\sigma}(\zeta)\right)}{T\left(r^{2}, F_{\sigma}(\zeta)\right)}
$$

an immediate consequence of the definition (11.6), it is clear from (11.7) that equality may hold in (2.15) for all nonintegral values of $\sigma=\lambda / 2$.

12. Functions of arbitrary order having two maximal Valiron deficiencies. It is well known that the order of a meromorphic function $f(z)$ places many stringent restrictions on the size of the Nevanlinna deficiencies $\delta(c, f)$ (cf. for example [2] and $[8$, Theorems $4.7,4.10,4.11,4.15])$. To indicate that such upper bounds do not exist for Valiron deficiencies, I prove

THEOREM 4. Let $\lambda$ be given $(0 \leqq \lambda \leqq+\infty)$. Then there exists an entire function of order $\lambda$, with negative zeros, such that

$$
\liminf _{r \rightarrow+\infty} \frac{N(r, 0)}{T(r)}=0
$$

and hence

$$
\Delta(0)=\Delta(\infty)=1
$$

Proof. Let $q$ be a given nonnegative integer, and denote by

$$
f(z)=\prod_{n=1}^{\infty} E\left(\frac{z}{a_{n}}, q\right)
$$

any canonical product of genus $q$ having only negative zeros. Then since $f(z)$ is entire, (5.5) implies

$$
\begin{array}{r}
T(r)=m(r, f) \geqq \int_{0}^{\infty} N(t, 0)\left[K_{q}(t, r, \beta)-K_{q}(t, r, b)\right] d t \\
\quad(0 \leqq b<\beta<\pi ; r \geqq 1)
\end{array}
$$


If $q$ is an even integer, choose $b=0$ and $\beta=\pi / 2(q+1)$. Then (12.3) and the definition (4.4) of $K_{q}$ yield

$$
T(r) \geqq \frac{1}{\pi} \int_{0}^{\infty} N(t, 0)\left(\frac{r}{t}\right)^{q+1} \frac{t}{(t+r)^{2}} d t \quad(r>0) .
$$

If $q$ is odd, put $b=\pi / 2(q+1)$ in (12.3) and let $\beta \rightarrow \pi-$. This leads to

$$
\begin{aligned}
T(r) & \geqq N(r, 0)-\int_{0}^{\infty} N(t, 0) K_{q}\left(t, r, \frac{\pi}{2(q+1)}\right) d t \\
& \geqq \frac{1}{\pi} \int_{0}^{\infty} N(t, 0)\left(\frac{r}{t}\right)^{q+1} \frac{t}{(t+r)^{2}} d t .
\end{aligned}
$$

Thus (12.4) and (12.5) imply, for any integer $q(\geqq 0)$,

$$
T(r) \geqq N(\sigma r, 0) \frac{1}{\pi} \int_{\sigma r}^{\infty}\left(\frac{r}{t}\right)^{q+1} \frac{t}{(t+r)^{2}} d t=K N(\sigma r, 0),
$$

where

$$
K=K(\sigma, q)=\frac{1}{\pi} \int_{\sigma}^{\infty} \frac{d u}{u^{q}(1+u)^{2}} \quad(\sigma>1)
$$

is a positive constant.

Hence the zeros of $f(z)$ satisfy

$$
K \frac{N(r, 0)}{T(r)} \leqq \frac{N(r, 0)}{N(\sigma r, 0)} .
$$

Let $\lambda$ be a given non-negative number, and choose the sequence $\left\{a_{n}\right\}$ to have exponent of convergence $\lambda$ and so that the counting function $n(r, 0)$ satisfies

$$
\liminf _{r \rightarrow+\infty} \frac{n(r, 0) \log r}{n(2 r, 0)}=0 \text {. }
$$

This is readily done by choosing the zeros $a_{n}$ so that $n(r, 0)$ is constant over sufficiently large intervals $t_{v} \leqq r \leqq T_{v}$.

Then the function $f(z)$ defined by (12.2) has order $\lambda$ (cf. [8, p. 27]), and in view of the obvious inequalities

$$
\begin{aligned}
N(r, 0) & \leqq n(r, 0)(\log r+\text { const. }) & \left(r \geqq r_{0}\right), \\
N(2 e r, 0) & \geqq n(2 r, 0) & (r \geqq 1),
\end{aligned}
$$

(12.7) implies, if $\sigma=2 e$,

$$
\liminf _{r \rightarrow+\infty} \frac{N(r, 0)}{N(\sigma r, 0)}=0
$$

Comparing (12.8) with (12.6), we obtain (12.1). 
In particular, it follows from (12.7) that there exist entire functions $f(z)$ of very slow growths which satisfy $(12.1)$, provided only that

$$
\limsup _{r \rightarrow+\infty} \frac{\log M(r, f)}{(\log r)^{2}}=+\infty
$$

holds. That the growth condition (12.9) is necessary for (12.1) is well known (cf. $[14$, p. 133]).

Examples of functions of infinite order for which (12.1) holds are provided by functions of the form $e^{g^{(z)}}$, where $g(z)$ is transcendental and entire.

\section{REFERENCES}

1. A. Edrei, The deficiencies of meromorphic functions of finite lower order, Duke Math. J. 31 (1964), 1-21.

2. - Sums of deficiencies of meromorphic functions, J. Analyse Math. 14 (1965), 79-107.

3. A. Edrei and W. H. J. Fuchs, On the growth of meromorphic functions with several deficient values, Trans. Amer. Math. Soc. 93 (1959), 292-328.

4. - The deficiencies of meromorphic functions of order less than one, Duke Math. J. 27 (1960), 233-249.

5. - On the maximum number of deficient values of certain classes of functions, AFOSR Report TN 60-402 (April 1960).

6. A. Edrei, W. H. J. Fuchs and S. Hellerstein, Radial distribution and deficiencies of the values of a meromorphic function, Pacific J. Math. 11 (1961), 135-151.

7. A. A. Gol'dberg, Distribution of values of meromorphic functions with separated zeros and poles, Soviet Math. Dokl. 2 (1961), 389-392.

8. W. K. Hayman, Meromorphic functions, Oxford Univ. Press, New York, 1964.

9. S. Hellerstein, On a class of meromorphic functions with deficient zeros and poles, Pacific J. Math. 13 (1963), 115-124.

10. R. Nevanlinna, Le théorème de Picard-Borel et la théorie des fonctions méromorphes, Gauthier-Villars Paris, 1930.

11. - Eindeutige analytische Funktionen, 2nd ed., Springer-Verlag, Berlin, 1953.

12. I. V. Ostrovskii, Some asymptotic properties of entire functions with negative zeros, Zap. Mat. Otd. Fiz.-Mat. Khar'kov Mat. Obšč. (4) 28 (1961), 23-32. (Russian)

13. G. Valiron, Sur les fonctions entières d'ordre fini et d'ordre nul, et en particulier les fonctions a correspondance réguliere, Ann. Fac. Sci. Univ. Toulouse (3) 5 (1913), 117-257.

14. - Lectures on the general theory of integral functions, Toulouse, 1923.

SYRACUSE UNIVERSITY,

SYRACUSE, New YoRK

IMPERIAL COLLEGR,

LONDON, ENGLAND 